\title{
Ionophore-mediated swelling of erythrocytes as a therapeutic mechanism in sickle cell disease
}

Athena C. Geisness1, Melissa Azul,,2* Dillon Williams, ${ }^{1}$ Hannah Szafraniec, Daniel C. De Souza, ${ }^{3,4,5}$ John M. Higgins, ${ }^{3,4,5}$ and David K. Wood ${ }^{1}$

${ }^{1}$ Department of Biomedical Engineering, University of Minnesota Twin Cities, Minneapolis, MN; ${ }^{2}$ Department of Pediatric and Adolescent Medicine, Division of Pediatric HematologyOncology, Mayo Clinic, Rochester, MN; ${ }^{3}$ Department of Systems Biology, Harvard Medical School, Boston, MA; ${ }^{4}$ Department of Pathology, Massachusetts General Hospital, Harvard Medical School, Boston, MA and ${ }^{5}$ Center for Systems Biology, Massachusetts General Hospital, Harvard Medical School, Boston, MA, USA

*ACG and MA contributed equally as co-first authors.

\section{Correspondence:}

David K. Wood

dkwood@umn.edu

Received: $\quad$ March 8, 2021.

Accepted: $\quad$ October 18, 2021

Pre published: October 28, 2021.

https://doi.org/10.3324 haematol.2021.278666

๑2022 Ferrata Storti Foundation

Haematologica material is under a license CC

$\mathrm{BY}-\mathrm{NC} @ \underset{\mathrm{ar}}{\mathrm{ar}} \mathrm{Ne}$

\begin{abstract}
Sickle cell disease (SCD) is characterized by sickle hemoglobin ( $\mathrm{HbS}$ ) which polymerizes under deoxygenated conditions to form a stiff, sickled erythrocyte. The dehydration of sickle erythrocytes increases intracellular HbS concentration and the propensity of erythrocyte sickling. Prevention of this mechanism may provide a target for potential SCD therapy investigation. Ionophores such as monensin can increase erythrocyte sodium permeability by facilitating its transmembrane transport, leading to osmotic swelling of the erythrocyte and decreased hemoglobin concentration. In this study, we treated 13 blood samples from patients with SCD with $10 \mathrm{nM}$ of monensin ex vivo. We measured changes in cell volume and hemoglobin concentration in response to monensin treatment, and we perfused treated blood samples through a microfluidic device that permits quantification of blood flow under controlled hypoxia. Monensin treatment led to increases in cell volume and reductions in hemoglobin concentration in most blood samples, though the degree of response varied across samples. Monensin-treated samples also demonstrated reduced blood flow impairment under hypoxic conditions relative to untreated controls. Moreover, there was a significant correlation between the improvement in blood flow and the decrease in hemoglobin concentration. Thus, our results demonstrate that a reduction in intracellular HbS concentration by osmotic swelling improves blood flow under hypoxic conditions. Although the toxicity of monensin will likely prevent it from being a viable clinical treatment, these results suggest that osmotic swelling should be investigated further as a potential mechanism for SCD therapy.
\end{abstract}

\section{Introduction}

Sickle cell disease (SCD) is an inherited blood disorder that affects approximately 100,000 Americans in the United States and decreases a patient's life expectancy by 30 years. ${ }^{1}$ The disease is caused by a genetic mutation in the $\beta$-globin gene which produces sickle hemoglobin ( $\mathrm{HbS}$ ). $\mathrm{HbS}$ can polymerize under deoxygenated conditions forming stiff, sickled red blood cells (SRBC). ${ }^{2}$ The presence of sRBC contributes to the key elements of SCD pathology: hemoglobin polymerization, endothelial dysfunction, sterile inflammation, leading to overall disruption of blood flow particularly in the microvasculature. ${ }^{3}$ These processes ultimately give rise to the vast clinical manifestations seen in SCD including vaso-occlusive episodes (VOE), acute chest syndrome, and stroke. The complex pathophysiology of SCD requires the development of treatments that target one or more of the molecular disease pathology mechanisms. ${ }^{4-5}$
Given that HbS polymerization is essential in the pathophysiology of SCD, treatments to prevent HbS polymerization continue to be an area of investigation for therapeutic development. ${ }^{6-8}$ Hydroxyurea, the first drug approved by the Food and Drug Administration (FDA) for the treatment of SCD, induces production of fetal hemoglobin ( $\mathrm{HbF})$, an anti-sickling hemoglobin. Though available for decades, patients treated with hydroxyurea experience variable clinical benefit and are subject to ongoing monitoring given its hematologic side effects. ${ }^{9}$ Recently, another antisickling agent, voxelotor, was approved by the FDA for the treatment of SCD. Voxelotor stabilizes the oxygenated form of HbS by increasing hemoglobin's oxygen affinity, preventing polymerization when exposed to deoxygenated conditions. ${ }^{10,11}$ Research is still ongoing to determine voxelotor's potential side effects and clinical benefit. In its phase III clinical trial, patients randomized to receiving voxelotor did experience an increase in hemoglobin after 
6 months of use, but they did not have a reduction in VOE." While treatments that inhibit polymerization such as voxelotor or hydroxyurea have been successful, therapeutics that target polymerization without affecting hemoglobin oxygen affinity or targeting the hematopoietic niche may provide similar clinical benefit without the side effects seen with these drugs.

An alternative mechanism to inhibit $\mathrm{HbS}$ polymerization is the reduction of intracellular $\mathrm{HbS}$ concentration within a SRBC. Small decreases in $\mathrm{HbS}$ concentration can lead to slower polymerization rates that are longer than SRBC capillary transit time. ${ }^{8,12}$ Previous studies to reduce $\mathrm{HbS}$ concentration include using antidiuretic hormone and a low sodium $(\mathrm{Na})$ diet to reduce plasma osmolality and $\mathrm{Na}$ concentration. This caused hypotonic swelling of SRBC and a reduction in mean cell hemoglobin concentration ( $\mathrm{MCHC}$ ), ultimately leading to decreased erythrocyte sickling observed in three patients. ${ }^{13}$ However, maintaining the necessary level of hyponatremia was impractical and results could not be reproduced in later studies. ${ }^{14,15}$ Rather than decreasing plasma $\mathrm{Na}$ and osmolality as a method to reduce $\mathrm{MCHC}$ and $\mathrm{HbS}$ polymerization, increasing intracellular $\mathrm{Na}$ and osmolality may be more feasible. This produces similar osmotic swelling effects and decreases $\mathrm{MCHC}$ without the difficulties of sustaining low plasma $\mathrm{Na}$ concentrations. In order to study this mechanism and its potential benefit in SCD, ionophores that increase the erythrocyte permeability to $\mathrm{Na}$, such as monensin, can be used to facilitate intracellular $\mathrm{Na}$ transport. Monensin selectively binds to $\mathrm{Na}^{+}$ions and facilitates its electrogenic transport across the erythrocyte membrane, creating an osmotic gradient and causing an influx of fluid intracellularly. ${ }^{16-18}$ Previous work in SRBC treated with monensin have demonstrated that monensin is effective at increasing mean corpuscular volume (MCV), decreasing $\mathrm{MCHC}$, and increasing deformability of SRBC. ${ }^{19-21}$ These studies provided a basis for understanding the molecular effects of monensin on RBC, however, they did not examine how these molecular changes impact the mechanics of RBC flow under physiologic conditions.

In this study, we use monensin as a model compound to investigate osmotic swelling to reduce $\mathrm{MCHC}$ as a potential mechanism for SCD therapy development. We aim to characterize the effects of SRBC osmotic swelling and reduced MCHC on SRBC rheologic oxygen dependence using a microfluidic device designed to recapitulate the physiological environment of the microvasculature. We compare the rheological response to hypoxia in our microfluidic device between blood samples treated with monensin and untreated controls. In order to further quantify its effect, we correlate $\mathrm{MCV}, \mathrm{MCHC}$, and the rheological response to hypoxia. By studying the effect monensin may have on rheology, we build upon previous monensin studies and are now able to better capture the complex pathophysio- logic changes in blood flow that occur with deoxygenated conditions in a physiologically relevant system, gaining a more comprehensive understanding of the potential therapeutic mechanism and its in vivo effects.

\section{Methods}

\section{Monensin treatment}

All study protocols were approved by the Institutional Review Board (IRB). In preparation for monensin treatment (Figure 1A), RBC were washed three times by centrifugation in Buffer A solution (104 mM NaCl, $32 \mathrm{mM} \mathrm{Na}{ }_{2} \mathrm{HPO}_{4}$, $8 \mathrm{mM} \mathrm{KH} 2 \mathrm{PO}_{4}, 5.5 \mathrm{mM}$ dextrose, $1 \mathrm{~g} / \mathrm{L}$ bovine serum albumin [BSA]; $\mathrm{pH} 7.4,305$ mOsm) with techniques previously published. ${ }^{18}$ Dextrose and BSA components of the Buffer A solution were added on the day of experiments. Samples were resuspended in Buffer A with $0.01 \% \mathrm{EtOH}$ and $10 \mathrm{nM}$ monensin (420701, BioLegend) to achieve 25\% hematocrit (hct) and incubated at $37^{\circ} \mathrm{C}$ for 12 hours (hrs). A concentration of $10 \mathrm{nM}$ monensin was chosen based on previous studies demonstrating optimal cellular effect without increased hemolysis. ${ }^{19}$ Preliminary research shows that incubation in Buffer A between 6 and 24 hrs limits RBC swelling in controls to less than $5 \% \mathrm{MCV}$. While the effect of $0.01 \% \mathrm{EtOH}$ on RBC has been previously studied to be insignificant, ${ }^{22-24}$ a control resuspended in Buffer $\mathrm{A}$ and $0.01 \% \mathrm{EtOH}$ was used for each sample in this study. After incubation, the sample was washed with phosphate-buffered saline (PBS) to remove extracellular monensin and resuspended in PBS to achieve $25 \%$ hct prior to rheology measurements. Details of blood sample collection, storage, and methods of obtaining laboratory values are provided in the Online Supplementary Appendix.

\section{Data collection and analysis}

Device design, fabrication, and experimental set up have been previously published ${ }^{24-26}$ and is detailed in the Online Supplementary Appendix. Continuous rheological data were captured using a high-speed camera (GS3-U3-23S6M-C, FLIR) at a frame rate of 500-600 FPS (frames per second) at 40x magnification. Blood flow velocity measurements were collected using a contrast detection algorithm developed in MATLAB based on the Kanade-Lucas-Tomasi algorithm. ${ }^{27-29}$ The velocity of thousands of contrasting points per frame were identified and averaged to obtain an average velocity per frame. Representative data in Figure $1 \mathrm{~B}$ demonstrates blood flow velocity under normoxic and hypoxic conditions for a control and monensin treated sample. Each sample was exposed to 1 minute of normoxia $(160 \mathrm{mmHg})$ and then 1 minute of hypoxia $(0 \mathrm{mmHg})$. This oxygenation-deoxygenation cycle was then repeated for a total of 3 cycles. Average steady state (SS) velocity at nor- 
moxia or hypoxia was determined by averaging the velocities of the three cycles at each oxygen tension for each sample. The average SS velocity value was used to determine two velocity metrics used for analysis: velocity response and recovery. Velocity response is defined by the difference between the average SS velocity at $160 \mathrm{mmHg}$ and $0 \mathrm{mmHg}$ oxygen. The response is normalized by the sample's average velocity at $160 \mathrm{mmHg}$ oxygen tension (Figure 1C) and indicates the magnitude of velocity reduc- tion during deoxygenation. Velocity recovery is defined by the difference in velocity response between treatment and the untreated control and indicates the change in velocity reduction during deoxygenation due to treatment.

\section{Statistical analysis}

A Wilcoxon signed-rank test is used to establish significant difference between control and treatment groups $(n=13)$. A Pearson's product moment correlation coefficient is

A

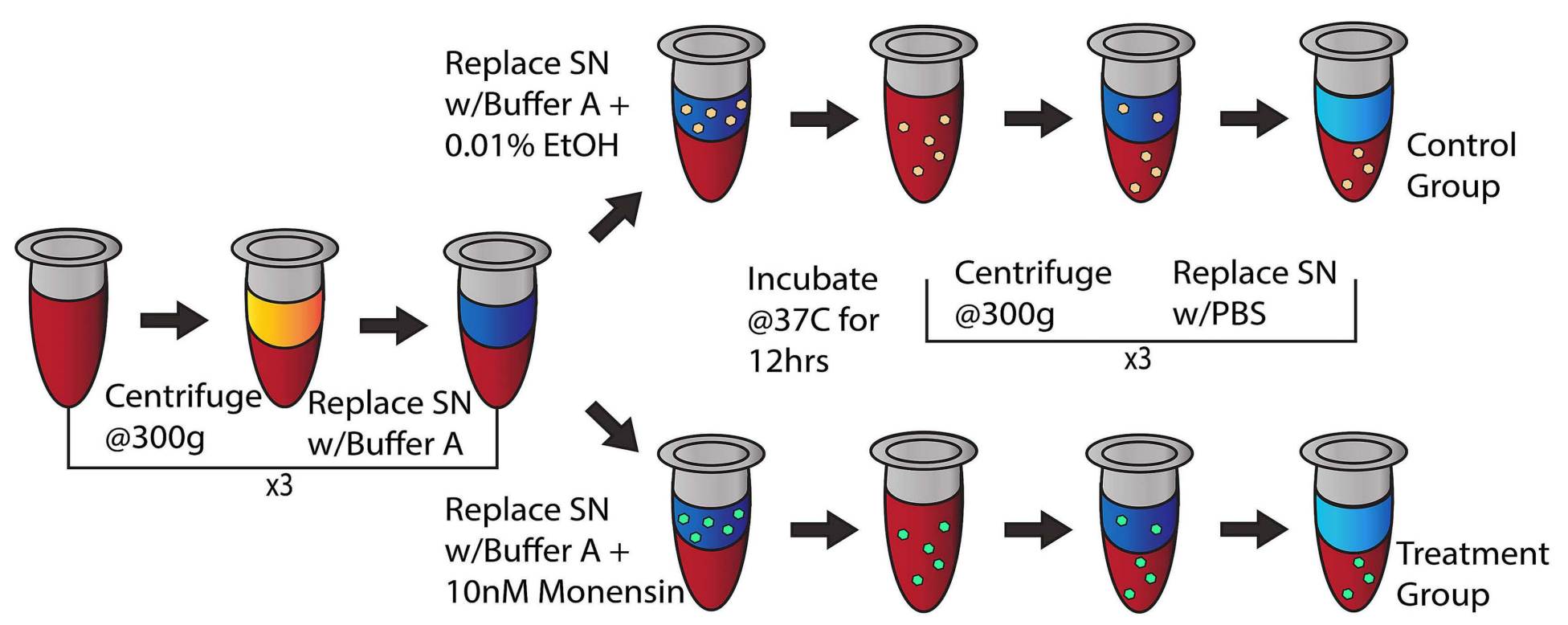

B
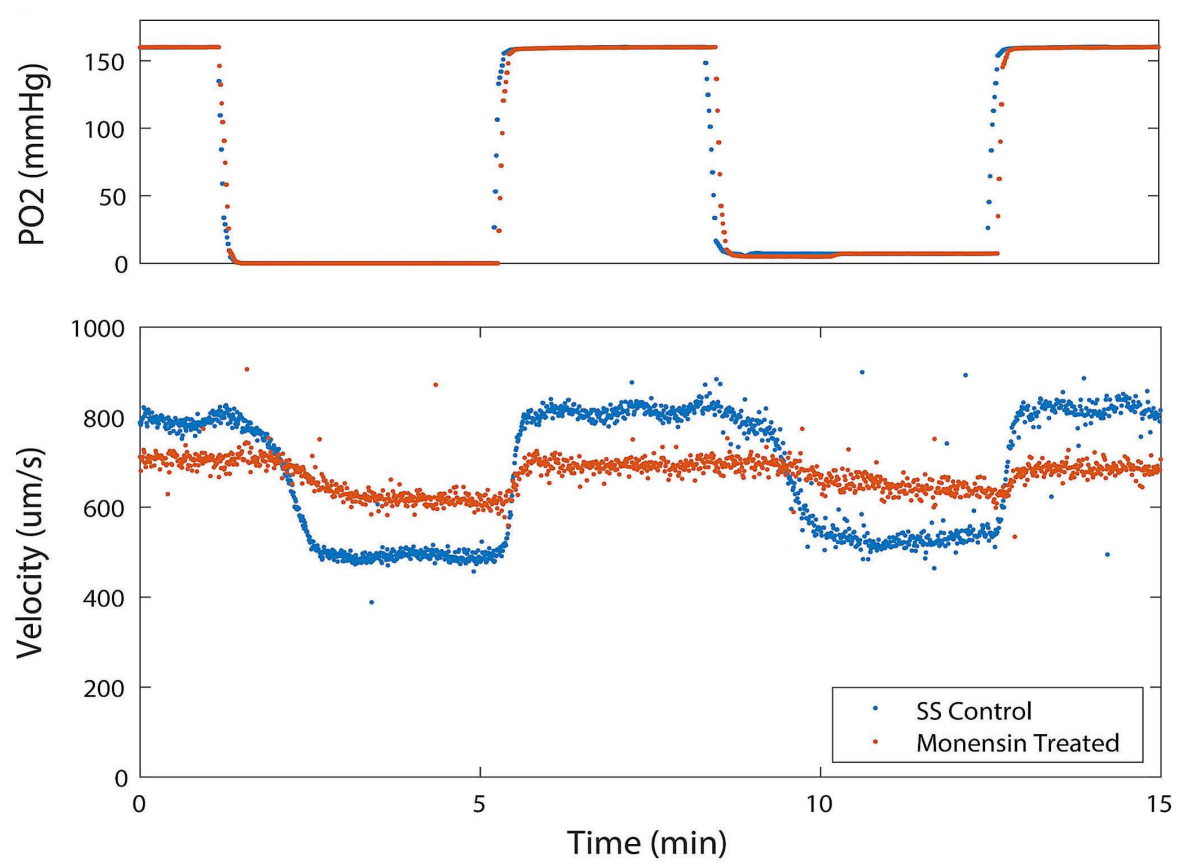

C

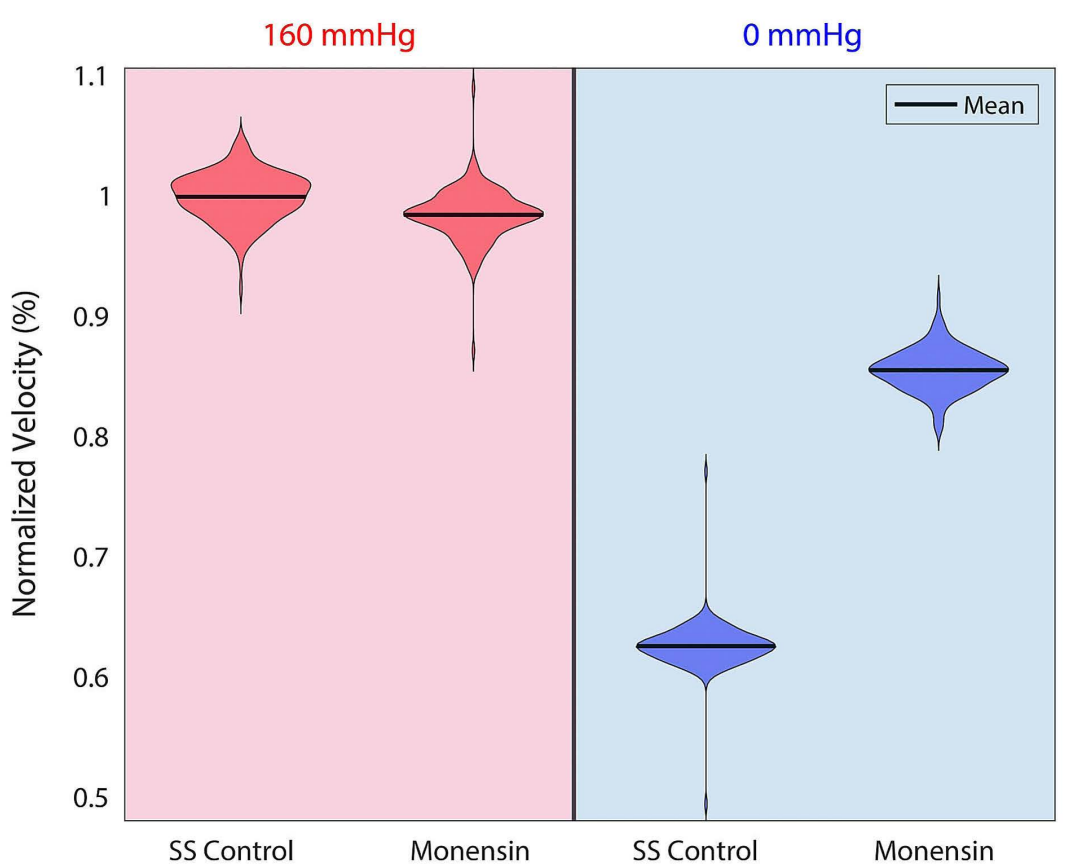

Figure 1. Data collection and analysis. An overview of methods of sample preparation, data collection, and defining rheological variables used throughout the study. (A) Schematic of the monensin treatment workflow. (B) Representative image of raw velocity data (below) as it relates to oxygen tension (above) from a single sickle cell disease (SCD) patient sample. In the bottom panel of (B), blood flow velocity is compared between monensin treatment (red) and the untreated control (blue). The average oxygenated shear rate during experiments was $355 \mathrm{~s}^{-1}$, within physiologic range for channel dimensions. ${ }^{46}$ In this sample, it appears that the velocity at normoxia of the monensin-treated condition is lower than that of the untreated condition. In order to address the differences in normoxic velocities between treatment conditions, conductance of all 13 samples was calculated to determine if additional variables were present contributing to normoxic velocities (Online Supplementary Figure S3). There were no significant differences in conductance at normoxia between treatment conditions in all samples, indicating velocity differences at normoxia were related to driving pressure. (C) The oxygenated $(160 \mathrm{mmHg})$ and deoxygenated $(0 \mathrm{mmHg})$ sections of the collected velocity data in (B), normalized by the average oxygenated steady state velocity for the representative sample. The representative single patient data in (C) demonstrates a 13\% velocity response for a monensin-treated sample and a $33 \%$ response for the untreated control. This corresponds to a velocity recovery of $20 \%$ after monensin treatment. Velocity response is calculated using the difference between oxygenated $(160 \mathrm{mmHg})$ and deoxygenated $(0 \mathrm{mmHg})$ velocities and velocity recovery is calculated by the difference in the control and monensin treated response. SN: supernatant; PBS: phosphate-buffered saline, SS: steady-state. 
used to describe the linear correlation $(n=13)$. Significance was defined by a $P \leq 0.05$.

\section{Results}

\section{Mean cell hemoglobin concentration strongly correlates with rheologic response to hypoxia}

A total of 13 samples from patients with SCD were obtained and used in experimentation. A summary of corresponding patient demographic, clinical, and baseline laboratory data are shown in Table 1. Several different sickle cell genotypes were included in the cohort. When oxygenation was decreased from $160 \mathrm{mmHg}$ to $0 \mathrm{mmHg}$, all untreated sickle samples responded with velocity reduction to a specific steady state velocity. When oxygen tension was restored back to $160 \mathrm{mmHg}$, blood flow velocity then increased and returned to its SS velocity prior to deoxygenation. Similar velocity response was replicated with repeated cycles of deoxygenation. The conductance of each sample at normoxia and hypoxia were calculated in each treatment condition to ensure non-significant differences in sample preparation and device variability between experiments (Online Supplementary Figure S2). In

Table 1. Patient demographics and laboratory values.

\begin{tabular}{|c|c|c|}
\hline Variable & \multicolumn{2}{|c|}{ N (13) } \\
\hline Genotype & \multirow{2}{*}{\multicolumn{2}{|c|}{7}} \\
\hline SS & & \\
\hline SC & \multicolumn{2}{|c|}{2} \\
\hline SBO & \multicolumn{2}{|c|}{3} \\
\hline SB+ & \multicolumn{2}{|c|}{1} \\
\hline \multicolumn{3}{|l|}{ Age (years) } \\
\hline Median (range, 23-42) & \multicolumn{2}{|c|}{27} \\
\hline \multicolumn{3}{|l|}{ Sex } \\
\hline Male & \multicolumn{2}{|c|}{6} \\
\hline Female & \multicolumn{2}{|c|}{7} \\
\hline \multicolumn{3}{|l|}{ Hydroxyurea } \\
\hline Yes & \multicolumn{2}{|c|}{12} \\
\hline \multirow[t]{2}{*}{ No } & \multicolumn{2}{|c|}{1} \\
\hline & Median & Range \\
\hline \multicolumn{3}{|l|}{ Clinical History } \\
\hline Transfusion* & 2 & $0-4$ \\
\hline Hospitalizations* & 1 & $0-2$ \\
\hline \multicolumn{3}{|l|}{ Laboratory Values } \\
\hline Hematocrit (\%) & 21.9 & $13.1-24.1$ \\
\hline MCV (fL) & 101.4 & $73.0-119.3$ \\
\hline $\mathrm{MCHC}(\%)$ & 33.3 & $29.7-36.2$ \\
\hline $\mathrm{HbA}(\%)$ & 15.8 & $7.2-46$ \\
\hline $\mathrm{HbS}(\%)$ & 67.8 & $38.2-86.5$ \\
\hline $\mathrm{HbF}(\%)$ & 7.5 & $0.5-21.6$ \\
\hline
\end{tabular}

* \# of transfusions or hospitalizations within the last year. N: number; MCV: mean corpuscular volume; MCHC: mean cell hemoglobin concentration; $\mathrm{Hb}$ : hemoglobin. contrast, oxygen-dependent velocity was not observed in healthy, AA, blood controls (Online Supplementary Figure S3A). In order to identify a parameter which may dictate a sample's velocity response to hypoxia, we first determined if $\mathrm{MCHC}$ and $\mathrm{MCV}$ were independent variables within the 13 untreated sickle samples. There was no correlation between MCV and MCHC (Figure 2A, $r=-0.008, P=0.982$ ), as $\mathrm{MCHC}$ is maintained between $30-36 \mathrm{~g} / \mathrm{dL}$ within $\mathrm{RBC}$ of varying sizes. These $\mathrm{MCHC}$ values are consistent with low to normal adult MCHC values typically seen in $\mathrm{SCD}^{30}$ and support previous work demonstrating that native MCV values have no correlation with native $\mathrm{MCHC}$ values in SCD. ${ }^{31}$ This supports that MCV and MCHC are likely independent variables and may individually influence the rheological response. In order to determine the influence of these variables on samples' velocity response, we compared MCV or MCHC with each sample's velocity response. There is a slight negative relationship between velocity response and MCV amongst the 13 untreated samples, though this correlation was not significant (Figure 2B, $r=-$ $0.13, P=0.660)$. There was, however, a significant positive relationship when correlating velocity response and $\mathrm{MCHC}$ (Figure 2C, $r=0.83, P=0.001$ ), as untreated samples with lower MCHC had smaller velocity responses when exposed to hypoxia. Collectively, this data corroborates previous work by others demonstrating that cell volume does not strongly correlate with the rheological response and rather it is hemoglobin concentration that is strongly correlated with sample blood flow response. ${ }^{32}$

\section{Monensin increases sickled red blood cells mean corpuscular volume, decreases mean cell hemoglobin concentration, and reduces hypoxia-induced polymerization}

In order to determine the effect of monensin on SRBC, MCV and MCHC values pre- and post-treatment were collected of all 13 SCD samples and shown in Figure 3A and B. Overall, the monensin-treated samples had significantly increased MCV (Figure 3A) and decreased MCHC (Figure 3B) when compared to the controls $(P<0.01)$. The significant effects in MCV and MCHC were also observed when treating three healthy, AA blood controls with monensin as well (Online Supplementary Figure S3B). Throughout the sample cohort, the effect of monensin on MCV and MCHC widely varied between samples. Some samples had large differences in MCV and MCHC after monensin treatment (sample ID: 11 and 12). Sample 11 experienced the largest change in both MCV and MCHC after treatment and was from a patient with $\mathrm{HbSB}^{+}$thalassemia. Sample 12 also experienced large changes in MCV and $\mathrm{MCHC}$ and came from a patient who had been recently transfused with a severe clinical phenotype. Others demonstrated only minor changes (sample ID: 3 and 7). Sample 3 came from a patient with SC disease and sample 7 was from a patient 
with $\mathrm{HbSB}^{\circ}$. Given that monensin drives cell swelling, measured by MCV, and decreases hemoglobin concentration, measured by $\mathrm{MCHC}$, these results reflect the degree in which the sample was affected by monensin.

Given the observed changes in MCV and $\mathrm{MCHC}$ with monensin treatment, to further demonstrate the mechanism of cell swelling to reduce sickle pathophysiology, we analyzed the morphology of cells from three additional sickle blood samples under shear flow and controlled oxygen tension using a previously published microfluidic chip. ${ }^{33} \mathrm{~A}$ full description of the device and the methodology as well as the samples' baseline hematological laboratory data can be found in the Online Supplementary Appendix (Online Supplementary Table S1). Monensin reduced the fraction of cells containing polymer when exposed to hypoxic oxygen tensions in all samples (Online Supplementary Figure S4). However, similar to the effect observed on MCV and $\mathrm{MCHC}$, the amount monensin reduced polymerized cells in hypoxia varied between samples.

\section{Monensin treatment improves rheological response to hypoxia}

In order to quantify the effect of monensin on SRBC blood flow velocity in a hypoxic environment, we examined the velocity response to $0 \mathrm{mmHg}$ oxygen tension between treated and untreated samples for the 13 SCD blood samples. In monensin-treated samples, there was a significant decrease in velocity response with deoxygenation compared to that of untreated controls ( $P<0.01$, Figure $3 C)$, indicating the efficacy of monensin in decreasing SRBC sensitivity to hypoxia. However, there was variability in the degree of response to monensin treatment across all samples. For example, in sample ID 11, monensin treatment eliminated almost all blood flow velocity oxygen dependence demonstrated by no velocity response to hypoxia compared to a $40 \%$ response in the control. This contrasts with sample ID 3 and 5, where there was no monensin effect on blood flow velocity response when compared to the untreated control. In AA samples, there was no change in velocity response between monensintreated and untreated controls, despite the significant changes in MCV and $\mathrm{MCHC}$ after monensin treatment (Online Supplementary Figure S3).

\section{Reduction in mean cell hemoglobin correlates with improved rheologic response to hypoxia}

In order o objectively determine whether the magnitude of the monensin-induced changes to MCHC or MCV affects the magnitude of change in velocity response to hypoxia, we compared the linear correlation between $\mathrm{MCV}$ or $\mathrm{MCHC}$ change induced by monensin and the velocity recovery of each sample. By using the absolute change in MCV and

A

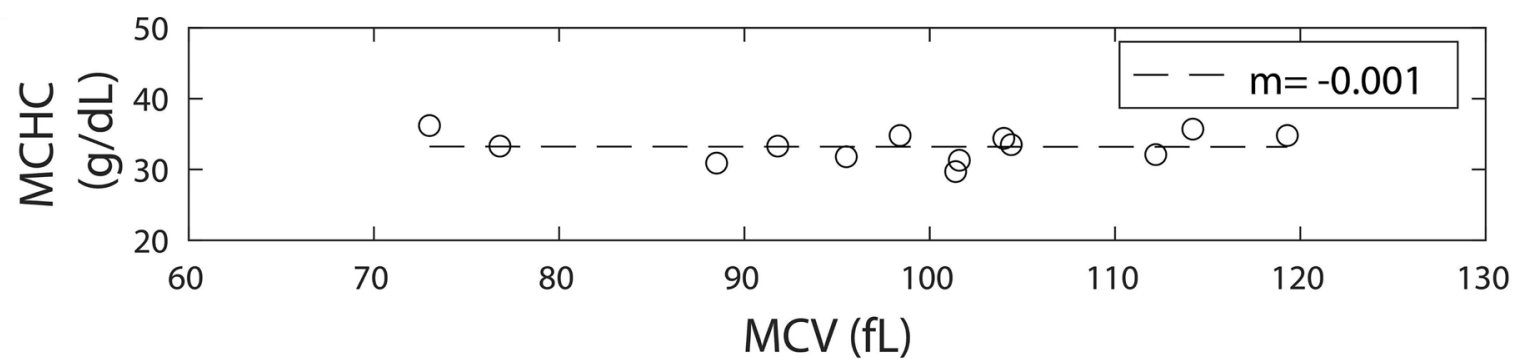

B

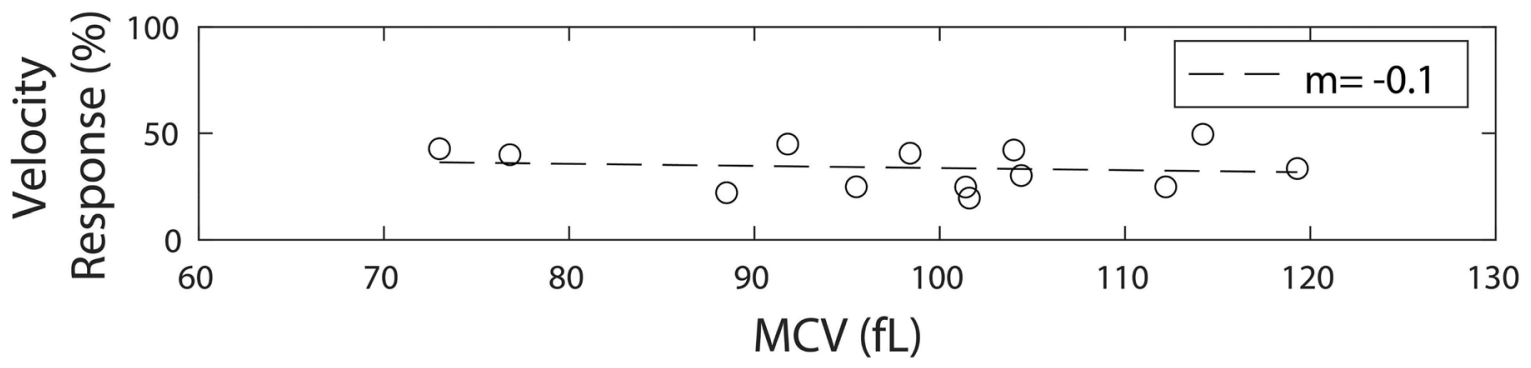

C

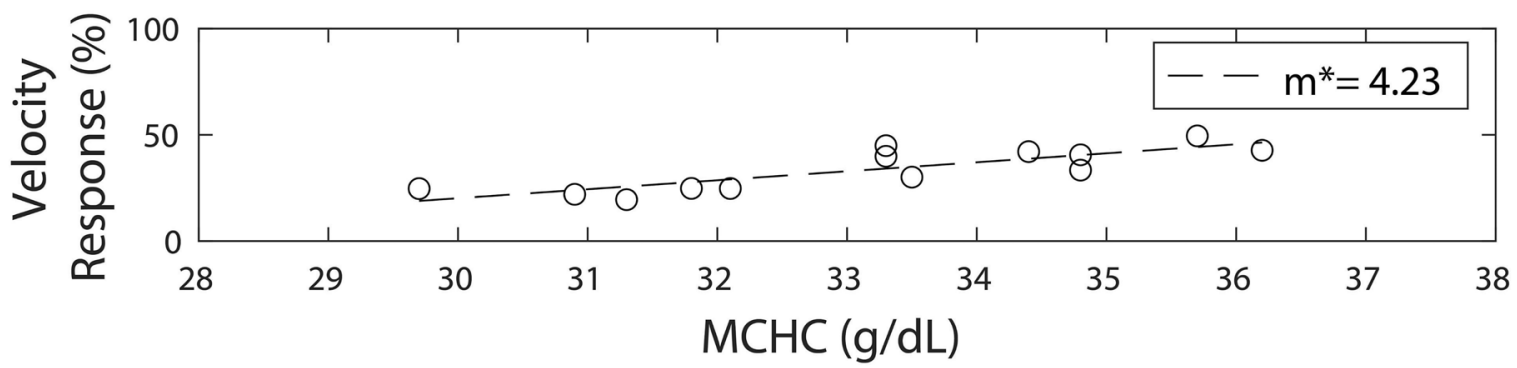

Figure 2. Dependent parameter analysis. Correlative data from 13 untreated sickle cell disease (SCD) samples to determine the relationship, if any, between mean corpuscular volume (MCV) and mean cell hemoglobin concentration (MCHC) and each variable's relation to sample velocity response. A Pearson correlation coefficient analysis was used to determine the strength of the linear relationship and a two-tailed analysis of the Pearson coefficient was used to determine significance of the correlation. (A) No correlation was identified between MCV and MCHC ( $r=-0.008, P=0.982$ ), establishing MCV and MCHC as independent variables. (B) MCV as it relates to velocity response. No correlation was identified between MCV values and velocity response $(r=-0.13, P=0.660)$. A slope of -0.001 and -0.1 are found in $(A)$ and (B) respectively. The slope for these figures is provided for clarification of the scale for (A and $B)$. (C) $M C H C$ and velocity response had a significant correlation $(r=0.83, P<0.001)$. 

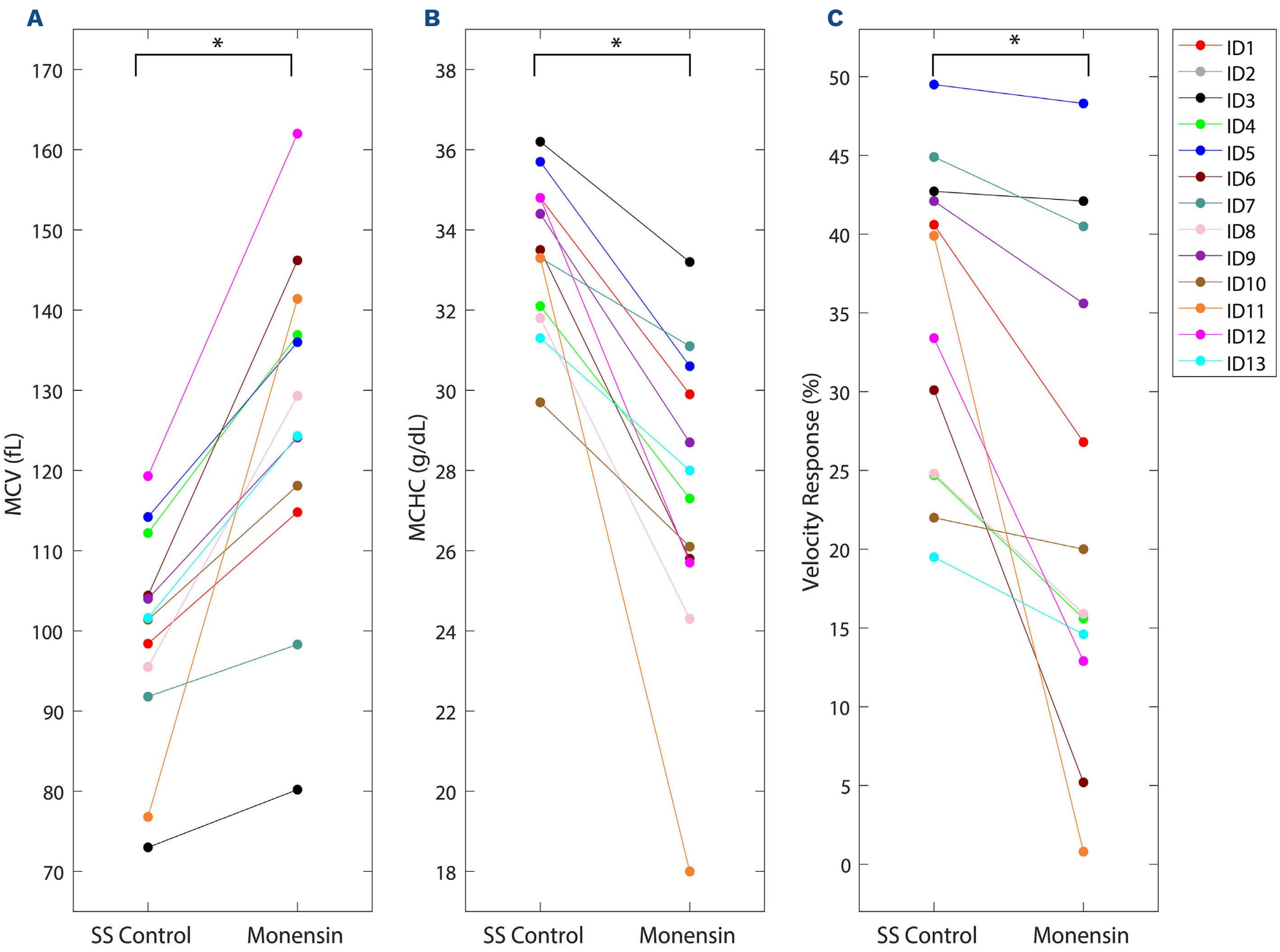

Figure 3. Monensin treatment efficacy and variability. Summary data of monensin treatment. (A and B) The effect of monensin on mean cell hemoglobin concentration $(\mathrm{MCHC})$ and mean corpuscular volume (MCV). The monensin-treated group had significantly higher MCV and lower MCHC compared to the control group. (C) There was a significantly lower velocity response in the monensin-treated group compared to that in the control group. Significance between control and monensin-treated groups was determined using a Wilcoxon signed-rank test and indicated by the asterisks (*) denoting $P<0.01$. Sample ID represents the de-identified patient ID corresponding to the sample. Error bars indicate the standard deviation in velocity response over 3 oxygenation/deoxygenations cycles. SS: steady state.

$\mathrm{MCHC}$, the analysis removes the variability of each sample's initial MCV and MCHC and controls for patients' baseline heterogenous clinical severity. First, we ensured correlation between $\mathrm{MCV}$ increases and $\mathrm{MCHC}$ decreases in the monensin-treated samples. In Figure 4A, there was a significant positive correlation between MCV change and $\mathrm{MCHC}$ change $(r=0.91, P<0.001)$, in that large MCV increases due to monensin corresponded with large $\mathrm{MCHC}$ reductions. This demonstrates cell swelling is an effective method to decrease MCHC. When comparing the degree in which MCV was increased by monensin and velocity recovery we found a significant positive correlation (Figure $4 \mathrm{~B}, \mathrm{r}=0.87, P<0.001)$. A more significant positive relationship is seen when comparing the degree to which monensin decreased $\mathrm{MCHC}$ and sample recovery (Figure 4C, $r=0.96, P<0.001)$, in that the largest improvements in sample velocity response to hypoxia correlated with larger reductions in $\mathrm{MCHC}$. These relationships reveal that the degree to which monensin affects sample sensitivity to hypoxia is strongly dependent on the degree to which the $\mathrm{MCHC}$ is reduced.

\section{Discussion}

In this study, we examined osmotic cell swelling to decrease intracellular $\mathrm{HbS}$ concentration as a potential mechanism to be targeted for future therapeutic development in SCD. We used a model $\mathrm{Na}$ ionophore compound, monensin, to treat SCD blood samples ex vivo. The samples were exposed to hypoxic conditions in a microfluidic device while blood flow was quantified. Though 
A

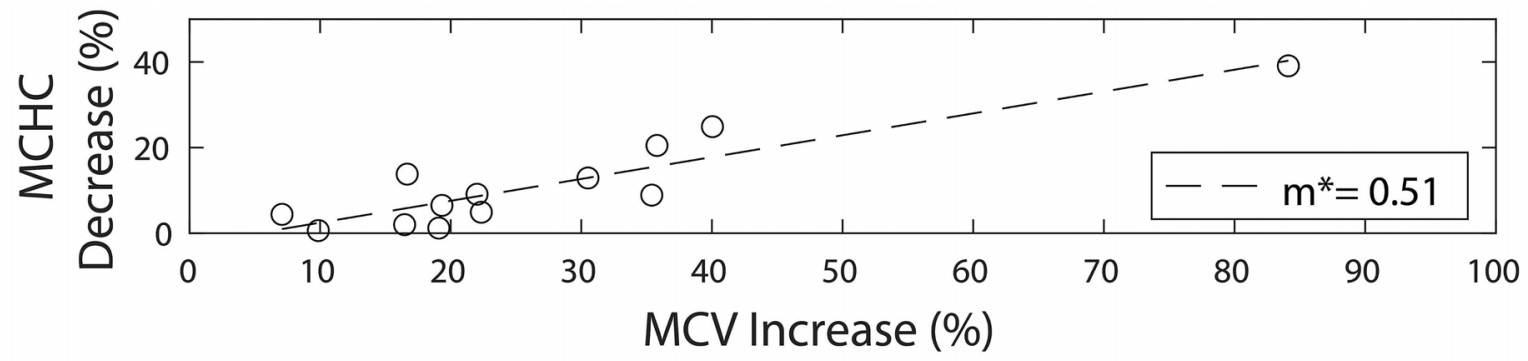

B
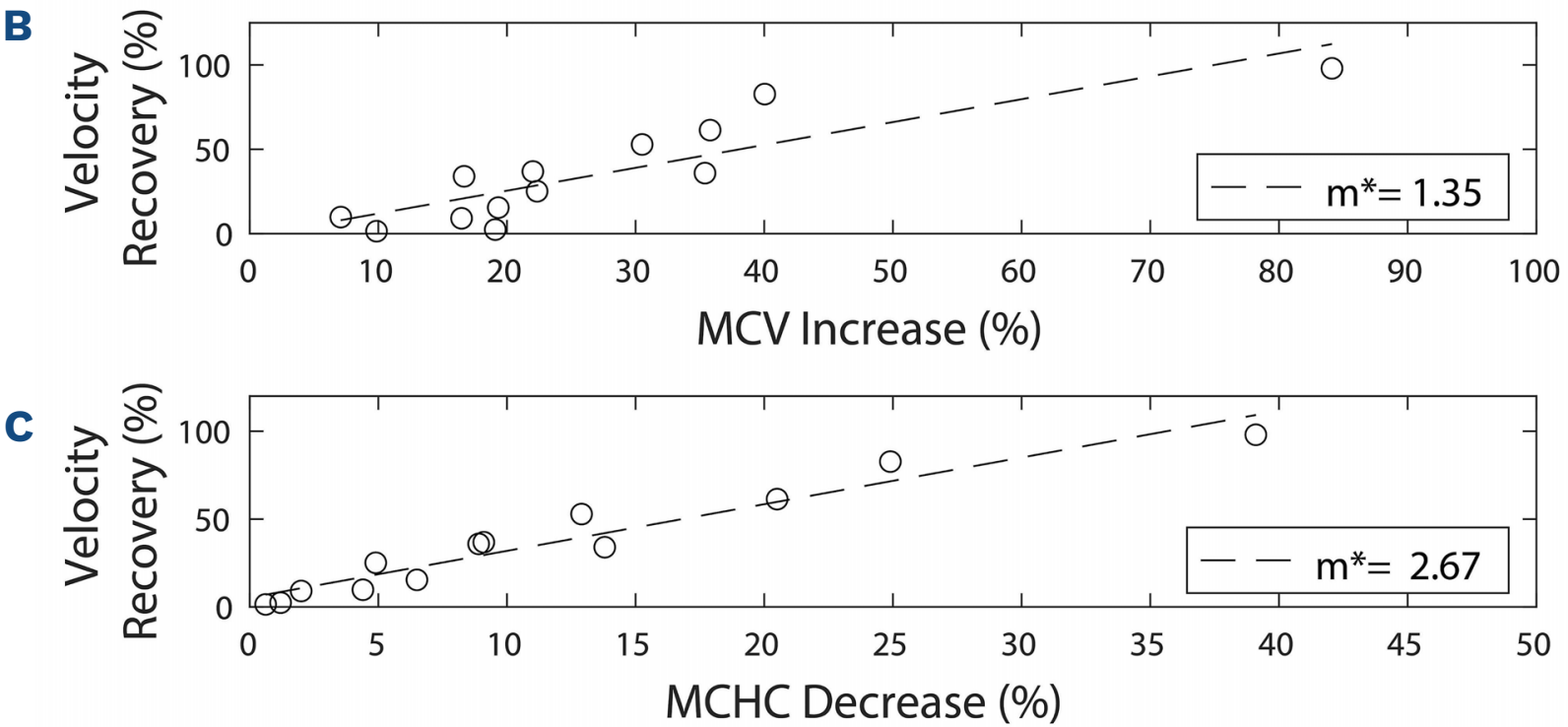

Figure 4. Linear correlation analysis. In order to determine which effect of monensin was driving sample velocity recovery, a correlation analysis to define the relationship between velocity recovery and monensin-induced mean corpuscular volume $(\mathrm{MCV})$ or mean cell hemoglobin concentration $(\mathrm{MCHC})$ change was completed. A Pearson correlation coefficient analysis was conducted to determine the strength of the linear relationship. (A) Change in MCV and change in MCHC was significantly positively correlated $(r=0.91, P<0.001)$. (B) Velocity recovery and $\mathrm{MCV}$ change also were positively significantly correlated ( $r=0.87$, $P<0.001)$. (C) The strongest correlation was found between velocity recovery and MCHC change $(r=0.96, P<0.01)$.

previous studies conducted in the early 1980's demonstrated monensin's ability to decrease sRBC MCHC, ${ }^{19}$ monensin's impact on dynamic blood flow, particularly in hypoxic environments, was not explored. Furthermore, deformability measurements were made through ektacytometry ${ }^{19}$ which may allude to improved rheology, however, monensin's global effect on blood flow was not directly measured. Additionally, more recent research reports ektacytometry deformability measurements are unreliable in predicting the ability of RBC perfusion of a microvascular network. ${ }^{34}$ Given that $\mathrm{MCHC}$ reduction was initially hypothesized to reduce polymerization and decrease vasoocclusion, investigating monensin's effect on dynamic flow rheology is critical to understanding whether the mechanism has a role in future therapeutic development. Using our microfluidic platform, we were able to observe and quantify monensin's effect of reduced MCHC on blood flow by measuring rheologic variables in a dynamic, physiologically relevant system. In our study, we not only found that monensin decreased $\mathrm{MCHC}$, but we also report that it significantly decreased the sensitivity of SCD blood flow to hypoxia compared to controls. The velocity recovery with monensin treatment varied between samples which correlated to the variation in monensin-induced changes in $\mathrm{MCHC}$. Through the rheological measurements obtained in this study, we provide both novel insight into the capability of this mechanism in the prevention of vaso-occlusion but also provide findings to suggest patient response variability.
While osmotic swelling and increases in MCV are the primary effects of the ionophore treatment, we found that the magnitude of $\mathrm{MCHC}$ reduction is the primary parameter modifying blood flow response to hypoxia. This is demonstrated by the significant correlation found between reduction in $\mathrm{MCHC}$ and reduction in velocity response (Figure 4C). When comparing response with MCV, the relationship is not as strongly correlated (Figure 4B). This corroborates existing studies which demonstrate that polymerization rates are extremely dependent on $\mathrm{HbS}$ concentration..$^{6-7}$ Additionally, we found that in the four samples that demonstrated insignificant change in velocity response when treated with monensin compared to controls (sample ID: $3,5,7$, and 10), there was less than a $5 \% \mathrm{MCHC}$ decrease. It is unclear what caused the observed patient variability in $\mathrm{MCV} / \mathrm{MCHC}$ response to monensin, however our data suggests that decreasing the MCHC by $5 \%$ has a significant impact on the sample's blood flow in hypoxia. Future studies that examine reduction of MCHC as a mechanism for SCD treatment should use a minimal threshold in $\mathrm{MCHC}$ reduction to guide drug efficacy.

This study investigated the mechanism of osmotic swelling to decrease intracellular $\mathrm{MCHC}$ thereby decreasing $\mathrm{HbS}$ polymerization. Similarly, previous SCD drug trials have focused on decreasing intracellular $\mathrm{MCHC}$ by inhibiting ion channels that are involved in the pathologic dehydration of sRBC. Clotrimazole, an inhibitor of the Gardos channel 
which contributes to SRBC water loss, demonstrated reduced SRBC dehydration, decreased $\mathrm{MCHC}$, and mild improvements in hemoglobin when taken by five SCD patients. ${ }^{35,36}$ Patients on Senicapoc, a similar Gardos channel inhibitor, also experienced significant increases in hemoglobin and hematocrit in a phase II clinical trial. Despite these promising results, studies involving Senicapoc were terminated early due to limited efficacy when no significant improvement in the rate of VOE were seen in those taking Senicapoc compared to those on placebo. ${ }^{37}$ While reductions in $\mathrm{MCHC}$ may correlate with reductions in polymerization-induced hemolysis and increased hemoglobin, it does not appear to be as correlated to frequency of VOE and implies that hemolysis and vaso-occlusion are distinct, yet intertwined, pathologic mechanisms. Further, although reductions in $\mathrm{MCHC}$ have been shown to decrease rigidity and stiffness of SRBC, ${ }^{38}$ over-swelling of SRBC by exposure to hypotonic solutions has also led to increased vaso-occlusions in in vitro models. ${ }^{39}$ Therefore, while the mechanism of osmotic swelling has been beneficial to distinct aspects of SCD pathology, it may not be effective or potentially problematic. Given that previous compounds have attempted to exploit a similar mechanism tested in this study but have ultimately proven unsuccessful due to a lack of reduction in VOE, the ability to determine how a compound affects blood flow, particularly in deoxygenated conditions like that of a VOE, is important in predicting its potential clinical success. Therapeutics that demonstrate improvement in overall blood rheology rather than on a single SCD pathophysiologic mechanism are likely to provide more benefit in reducing VOE frequency. By using microfluidic technology in this study, we can characterize the effect of monensin on velocity response and demonstrate that the mechanism improves rheological behavior. Due to its recognized narrow therapeutic window, use of monensin in veterinary medicine has led to several toxicities and accidental death, and therefore would not be an ideal agent for human study. ${ }^{40}$ However, this study motivates development of other compounds that may have similar osmotic swelling effects on erythrocytes for SCD treatment. ${ }^{41}$

A potential concerning side effect of this treatment mechanism includes increased blood viscosity due to increased cell volume and hct. With increased viscosity, the potential for VOE is amplified. ${ }^{41}$ Viscosity, however, is dependent on several factors such as hct, RBC deformability and aggregation. ${ }^{41,42}$ Previous studies demonstrate with decreased MCHC, SRBC deformability increases. ${ }^{43}$ Additionally, it has been reported that cell stiffness has a greater influence on blood viscosity than hct ${ }^{41}$ and as previously mentioned, monensin treatment has demonstrated increased SRBC deformability when compared to untreated controls. ${ }^{8}$ Patients treated with hydroxyurea also experience an increase in MCV without association of worsening outcomes. ${ }^{44}$ Additionally, although our study does not control for post-monensin increase in hct, we still demonstrate improved rheological behavior. Therefore, while osmotic SRBC swelling may increase viscosity, RBC deformability appears to be stronger determining factor in overall blood viscosity.

Our study is limited by primarily demonstrating correlative relationships with little experimentation on causation. However, previous work demonstrating increases in RBC deformability and decreases in SRBC fraction with monensin treatment, support the reduced rheological response to hypoxia observed in this study. While we demonstrate that decreasing MCHC by erythrocyte osmotic swelling successfully reduces SRBC oxygen dependent flow, this study does not capture the complex biological interactions between the many other cellular components involved in the pathophysiology of SCD. For example, in our experiments, we used washed red cells rather than whole blood. Previous studies found that monensin had a reduced effect on SRBC in the presence of plasma and required significantly increased concentrations to replicate improved deformability, indicating that the drug likely binds across plasma constituents. ${ }^{19}$ While using patient plasma may be helpful in determining concentration of monensin to observe rheological improvement, this study was to demonstrate the efficacy of the mechanism of $\mathrm{MCHC}$ reduction to improve SRBC rheological behavior. Should further studies exploring this mechanism be conducted, or for future therapeutic development, agents that specifically target RBC Na permeability by RBC-specific cation channels or transport would be of priority to reduce effect on other potential cellular components. Additionally, the endothelium is particularly of interest given that increased RBC deformability has been shown to increase endothelial adhesion and may contribute to the occurrence of VOE and clinical severity. ${ }^{45,46}$ Further studies examining the effect of $\mathrm{MCHC}$ reduction on adhesion and inflammation are needed to assess the potential benefit of this therapeutic mechanism. As discovery of SCD pathophysiology reveals more intricate biological pathways and multifaceted systems simultaneously at play, the approach to treatment may require an equally multifaceted, multi-drug approach. By combining a therapy that reduces $\mathrm{MCHC}$ and polymerization with an anti-adhesion therapy, perhaps further benefit may be achieved. Studies are needed to determine the advantages of a multi-agent approach, however similar strategies have already been successful in HIV therapy, cardiology, and oncology.

\section{Conclusion}

The reduction of $\mathrm{MCHC}$ through osmotic swelling of a sickle erythrocyte can effectively decrease the rheological dependence on oxygenation. Blood flow velocity measurements within microfluidic channels, of physiologic dimen- 
sions, indicate a strong correlation between $\mathrm{MCHC}$ reduction and reduction of blood flow sensitivity to hypoxia. These relationships may indicate the potential efficacy of regulating $\mathrm{MCHC}$ as a targeted mechanism for SCD therapeutic development.

\section{Disclosures}

No conflicts of interest to disclose.

\section{Contributions}

ACG, MA, DW, HS, and DCD performed research and data analysis; $A C G, D W, H S, D C D, D K W$ designed the studies; $A C G$, $M A, D W, D C D, J M H$ and $D K W$ wrote the manuscript.

\section{Acknowledgments}

We would like to thank Chhaya Patel, Hasmukh Patel, and Yvonne Datta for assistance with blood sample collection according to IRB protocol 2006P000066/PHS and STUDYOOOOOO3.

\section{Funding}

Portions of this work were conducted in the Minnesota Nano Center, which is supported by the National Science Foundation through the National Nano Coordinated Infrastructure Network (NNCI) under Award Number ECCS-1542202. We would like to thank the National Heart Lung and Blood Institute for their support under grant no. HL132906.

\section{References}

1. Piel FB, Steinberg $M H$, Rees DC. Sickle cell disease. N Engl J Med. 2017;376(16):1561-1573.

2. Barabino GA, Platt MO, Kaul DK. Sickle cell biomechanics. Annu Rev Biomed Eng. 2010;12:345-367.

3. Sundd P, Gladwin MT, Novelli EM. Pathophysiology of sickle cell disease. Annu Rev Pathol. 2019;14:263-292.

4. Riley TR, Boss A, McClain D, Riley TT. Review of medication therapy for the prevention of sickle cell crisis. $\mathrm{P}$ T. 2018;43(7):417-437.

5. Matte A, Zorzi F, Mazzi F, Federti F, Olivieri O, Francheschi LD. New therapeutic options for the treatment of sickle cell disease. Mediterr J Hematol Infect Dis. 2019;11(1):e2019002.

6. Ferrone FA. Polymerization and sickle cell disease: a molecular view. Microcirculation. 2004;11(2):115-128.

7. Hofrichter J, Ross PD, Eation WA. Kinetics and mechanism of deoxyhemoglobin $S$ gelation: a new approach to understanding sickle cell disease. Proc Natl Acad Sci USA. 1974;71(12):4864-4868.

8. Eaton WA, Bunn HF. Treating sickle cell disease by targeting HbS polymerization. Blood. 2017;129(20):2719-2726.

9. McGann PT, Ware RE. Hydroxyurea for sickle cell anemia: what have we learned and what questions still remain? Curr Opin Hematol. 2011;18(3):158-165.

10. Oksenberg D, Dufu K, Patel MP, et al. GBT440 increases haemoglobin oxygen affinity, reduces sickling and prolongs RBC half-life in a murine model of sickle cell disease. $\mathrm{Br} \mathrm{J}$ Haematol. 2016;175(1):141-153.

11. Vichinsky E, Hoppe CC, Ataga Al, et al. A phase 3 randomized trial of Voxelotor in sickle cell disease. N Engl J Med. 2019;381(6):509-519.

12. Eaton WA, Hofrichter J. Hemoglobin $S$ gelation and sickle cell disease. Blood. 1987;70(5):1245-1266.

13. Rosa RM, Bierer BE, Thomas R, et al. A study of induced hyponatremia in the prevention and treatment of sickle-cell crisis. N Engl J Med. 1980;303(20):1138-1143.

14. Leary M, Abramson N. Induced hyponatremia for sickle-cell crisis. N Engl J Med. 1981;304(14):844-845.

15. Charache $S$, Walker WG. Failure of Desmopressin to lower serum sodium or prevent crisis in patients with sickle cell anemia. Blood. 1981;58(5):892-896.

16. Stillwell W. Chapter 19: Membrane Transport. An Introduction to Biological Membranes 2nd Edition. 2016;440-443.

17. Freedman JC. Chapter 4 - Ionophores in Planar Lipid Bilayers. Cell Physiology 4th Edition. 2012;61-66.

18. Aowicki D, Huczyński A. Structure and antimicrobial properties of monensin A and its derivatives: summary of the achievements. Biomed Res Int. 2013;2013:742149.

19. Clark MR, Mohandas N, Shohet SB. Hydration of sickle cells using the sodium ionophore monensin. A model for therapy. J Clin Invest. 1982;70(5):1074-1080.

20. Li Q, Henry ER, Hofrichter J, et al. Kinetic assay shows that increasing red cell volume could be a treatment for sickle cell disease. Proc Natl Acad Sci USA. 2017;114(5):E689-E696.

21. Lu L, Li Z, Li H, Li X, Vekilov PG, Karniadakis GE. Quantitative prediction of erythrocyte sickling for the development of advanced sickle cell therapies. Sci Adv. 2019;5(8):eaax3905.

22. Sonmez M, Ince HY, Yalcin O, et al. The effect of alcohols on red blood cell mechanical properties and membrane fluidity depends on their molecular size. PLoS One. 2013;8(9):e76579.

23. Lee S, Park H. Best-Popescu C, Jang S, Park Y. The effects of ethanol on the morphological and biochemical properties of individual human red blood cells. PLoS One. 2015;10(12):e0145327.

24. Wood DK, Soriano A, Mahadevan L, Higgins JM, Bhatia SN. A biophysical indicator of vaso-occlusive risk in sickle cell disease. Sci Transl Med. 2012;4(123):123ra26.

25. Lu X, Wood DK, Higgins JM. Deoxygenation reduces sickle cell blood flow at arterial oxygen tension. Biophys J. 2016;110(12):2751-2758.

26. Valdez JM, Datta YH, Higgins JM, Wood DK. A microfluidic platform for simultaneous quantification of oxygen-dependent viscosity and shear thinning in sickle cell blood. APL Bioeng. 2019;3(4):046102.

27. Lucas BD, Kanade T. An iterative image registration technique with an application to stereo vision. IJCAI. 1981;674-679.

28. Tomasi C, Kanade T. Detection and tracking of point features. Carnegie Mellon University Technical Report CMU-CS-91-132. 1999 April.

29. Shi J, Tomasi C. Good Features to Track. IEEE Conference on Computer Vision and Pattern Recognition. 1994;593-600.

30. MCHC Blood Test - Low, High, What does it Mean. actforlibraries.org. 2017.

31. Serjeant G, Serjeant B, Stephens A, et al. Determinants of haemoglobin level in steady-state homozygous sickle cell disease. Br J Haematol. 1996:92(1):143-149.

32. Kaul DK, Liu XD. Rate of deoxygenation modulates rheologic behavior of sickle red blood cells at a given mean corpuscular hemoglobin concentration. Clin Hemorheol Microcirc. 1999;21(2):125-135. 
33. Di Caprio G, Schonbrun E, Goncalves BP, Valdez JM, Wood DK, Higgins JM. High-throughput assessment of hemoglobin polymer in single red blood cells from sickle cell patients under controlled oxygen tension. Proc Natl Acad Sci USA. 2019;116(50):25236-25242.

34. Sosa JM, Nielsen ND, Vignes SM, Tanya GC, Shevkoplyas SS. The relationship between red blood cell deformability metrics and perfusion of an artificial microvascular network. Clin Hemorheol Microcirc. 2014;57(3):275-228.

35. Brugnara C, Gee B, Armsby CC, et al. Therapy with oral clotrimazole induces inhibition of the Gardos channel and reduction of erythrocyte dehydration in patients with sickle cell disease. J Clin Invest. 1996;97(5):1227-1234.

36. Brugnara C. Sickle cell dehydration: pathophysiology and therapeutic applications. Clin Hemorheol Microcirc. 2018;68(23):187-204.

37. Ataga KI, Reid M, Ballas SK, et al. Improvements in haemolysis and indicators of erythrocyte survival do not correlate with acute vaso-occlusive crises in patients with sickle cell disease: a phase III randomized, placebo-controlled, double-blind study of the Gardos channel blocker Senicapoc (ICA-17043). Br J Haematol. 2011;153(1):92-104.

38. Evans E, Mohandas N, Leung A. Static and dynamic rigidities of normal and sickle erythrocytes. Major influence of cell hemoglobin concentration. J Clin Invest. 1984;73(2):477-488.
39. Carden MA, Fay ME, Lu X, et al. Extracellular fluid tonicity impacts sickle red blood cell deformability and adhesion. Blood. 2017;130(24):2654-2663.

40. Connes P, Alexy T, Detterich, J, Romana M, Hardy-Dessources MD, Ballas SK. The role of blood rheology in sickle cell disease. Blood Rev. 2016;30(2):111-118.

41. Novilla MN. The veterinary importance of the toxic syndrome induced by ionophores. Vet Hum Toxicol. 1992;34(1):66-70.

42. Nader E, Skinner S, Romana M, et al. Blood rheology: key parameters, impact on blood flow, role in sickle cell disease and effects of exercise. Front Physiol. 2019;10:1329.

43. Chien S. Determinants of blood viscosity and red cell deformability. Scand J Clin Lab Invest. 1981;156:7-12.

44. Ferster A, Vermylen C, Cornu G, et al. Hydroxyurea for treatment of severe sickle cell anemia: a pediatric clinical trial. Blood. 1996;88(6):1960-1964.

45. Hebbel RP, Moldow CF, Steinberg MH. Modulation of erythrocyte-endothelial interactions and the vaso-occlusive severity of sickling disorders. Blood. 1981;58(5):947-952.

46. Kaul DK, Chen D, Zhan J. Adhesion of sickle cells to vascular endothelium is critically dependent on changes in density and shape of the cells. Blood. 1994;83(10):3006-3007.

47. Sakariassen KS, Orning L, Turitto VT. The impact of blood shear rate on arterial thrombus formation. Future Sci OA. 2015;1(4):FSO30. 